\title{
Population Structure of Pseudocercospora fijiensis in Costa Rica Reveals Shared Haplotype Diversity with Southeast Asian Populations
}

\author{
Amanda Saville, Melodi Charles, Suchitra Chavan, Miguel Muñoz, Luis Gómez-Alpizar, and Jean Beagle Ristaino ${ }^{\dagger}$
}

First, second, third, and sixth authors: Department of Plant Pathology, North Carolina State University, Raleigh 27695; third author: Department of Genetics and Biochemistry, Clemson University, Clemson, SC 29631; fourth author: Dole Standard Fruits, SA, Tropical Research Department, Rio Frio, Costa Rica; and fifth author: Agronomic Research Center, University of Costa Rica, San Pedro, Montes de Oca, Costa Rica.

Accepted for publication 28 July 2017.

\begin{abstract}
Pseudocercospora fijiensis is the causal pathogen of black Sigatoka, a devastating disease of banana that can cause 20 to $80 \%$ yield loss in the absence of fungicides in banana crops. The genetic structure of populations of $P$. fijiensis in Costa Rica was examined and compared with Honduran and global populations to better understand migration patterns and inform management strategies. In total, 118 isolates of P. fijiensis collected from Costa Rica and Honduras from 2010 to 2014 were analyzed using multilocus genotyping of six loci and compared with a previously published global dataset of populations of $P$. fijiensis.

The Costa Rican and Honduran populations shared haplotype diversity with haplotypes from Southeast Asia, Oceania, and the Americas but not Africa for all but one of the six loci studied. Gene flow and shared haplotype diversity was found in Honduran and Costa Rican populations of the pathogen. The data indicate that the haplotypic diversity observed in Costa Rican populations of P. fijiensis is derived from dispersal from initial outbreak sources in Honduras and admixtures between genetically differentiated sources from Southeast Asia, Oceania, and the Americas.
\end{abstract}

Banana and plantain are the world's fourth most valuable food after rice, wheat, and maize. Only approximately $10 \%$ of the total production of banana fruit reaches international markets, while the rest are consumed locally in tropical regions as an important staple food (Ploetz 2001). Production of banana worldwide is affected due to black Sigatoka, or black leaf streak disease, caused by the fungal pathogen Pseudocercospora fijiensis (M. Morelet) Deighton (Teleomorph Mycosphaerella fijiensis M. Morelet) (Deighton 1976). In the absence of fungicides, black Sigatoka can cause $>80 \%$ yield loss in some areas (Marciel Cordeiro and Pires de Matos 2003). Effective management of black Sigatoka can require $>70$ fungicide applications in a year, increasing expenses significantly and making banana farming difficult for small shareholder and subsistence farmers (Abadie et al. 2009; Ploetz 2001).

Black Sigatoka was first described in Sigatoka Valley on Vitu Levu, Fiji, in 1963, although some sources suggest it was detected as early as 1927 in Taiwan (Churchill 2011; Stover 1978). Black sigatoka has spread to almost all banana production regions of the world, in many places replacing the less aggressive yellow Sigatoka, caused by the sister species M. musicola R. Leach ex J. L. Mulder (Halkett et al. 2010; Johanson 1995; Robert et al. 2012a). The large geographical range expansion of this emerging plant disease in just over 50 years is a classic example of a bioinvasion that threatens an important food crop and represents a threat to food security in tropical regions (Anderson et al. 2004; Desprez-Loustau et al. 2007). To reduce expensive and hazardous use of fungicides, efforts directed toward breeding for resistance against black Sigatoka have been implemented since 1959, when the United Fruit Company (UFC) established a breeding program in La Lima, Honduras. This program was later transferred to the Fundación Hondureña de Investigación Agrícola (FHIA) (Ploetz

†Corresponding author: J. B. Ristaino; E-mail: Jean_Ristaino@ncsu.edu

*The $e$-Xtra logo stands for "electronic extra" and indicates that three supplementary figures and five supplementary tables are published online.

(C) 2017 The American Phytopathological Society
2001). Other breeding programs have also been established, such as those at the International Institute of Tropical Agriculture in Nigeria in 1988 (Etebu and Young-Harry 2011). The results of these programs have generated banana and plantain cultivars with resistance to black Sigatoka; however, the banana cultivars developed have yet to be adopted beyond local markets (Ortiz and Vuylsteke 1994; Ploetz 2001). The selection and deployment of durable, resistant host cultivars requires an understanding of the pathogen population biology, including knowledge of genotypes, pathogen fitness, recombination, mutation, and migration.

The genetic variation of populations of $P$. fijiensis has been studied by multiple groups (Carlier et al. 1996; Halkett et al. 2010; Rieux et al. 2013; Rivas et al. 2004; Robert et al. 2012a). Most recently, Robert et al. (2012a) examined the introduction routes of $P$. fijiensis over four continents using 735 isolates from 37 countries. The study was conducted using 21 microsatellites and 8 sequencebased markers and confirmed the occurrence of genetic bottlenecks outside of Southeast Asia. By contrast, there were high levels of private allelic richness and a large number of private haplotypes in Southeast Asia, suggesting a long history for P. fijiensis in the region. These data suggested a Southeast Asian origin for the pathogen, and described a potential scenario in which $P$. fijiensis coevolved with its banana host, whose center of origin is also Southeast Asia (Robert et al. 2012a). This coevolution is predicted to have occurred during domestication of the genus Musa L. The results suggest that $P$. fijiensis originally migrated from Papua New Guinea in Southeast Asia to Oceania and African regions through separate migration events, most likely through the transport of infected plant material. Similar migration events brought $P$. fijiensis to the Americas from Southeast Asia or Oceania. There was clear genetic differentiation between isolates from the Americas and Africa. The isolates from Africa formed an isolated, relatively homogenous cluster, suggesting a single introduction event from a single Southeast Asian source population around the South China Sea. Microsatellite and sequence analyses of the American populations showed the presence of admixture between different genetically differentiated sources from Southeast Asia and Oceania, 
also suggesting multiple introduction events (Robert et al. 2012a). The array of genetic tools now available, including multilocus genotyping, simple sequence repeats, and next-generation sequencing, will enable more rapid and expansive studies of populations of $P$. fijiensis globally to track emerging and new outbreaks of the disease.

P. fijiensis was first reported in the Western Hemisphere in Honduras in 1972 (Stover 1974). However, examinations of photographs from 1969 suggest that the disease may have been present but undetected as early as the mid-1960s (Stover and Dickson 1976). Since then, the pathogen has spread throughout bananaproducing regions in Central and South America and into Florida as well (Ploetz 2001).

In 2012, Costa Rica was the second largest exporter of banana in Central and South America, behind Ecuador, and exported 2.1 million tons of banana (FAO 2014). P. fijiensis was first reported in Costa Rica in 1979 and remains a significant threat to banana production on both large- and small-scale farms in the country. Although significant progress has been made in describing the global population structure of $P$. fijiensis, additional knowledge of the population structure of the pathogen in Costa Rica is limited and is required to better understand inoculum sources and inform management strategies (Robert et al. 2012a). Black Sigatoka on banana in Costa Rica is of particular interest, due to extensive monoculture of banana on expansive commercial farms.

Our objectives were to (i) Characterize the genetic structure of populations of $P$. fijiensis in Costa Rica, (ii) infer potential migration sources of pathogen inoculum based on a global dataset, and (iii) compare the population structure of $P$. fijiensis in Costa Rica to populations of $P$. fijiensis collected from the Honduran field where the first reported outbreaks occurred in Central America.

\section{MATERIALS AND METHODS}

Sampling and DNA Extraction. The pathogen was sampled from Costa Rica and Honduras between 2010 and 2014 to obtain isolates $(n=118)$. Collections were made from mature lesions found in the leaves of Musa spp. (Supplementary Table S1). The isolates were collected from sprayed or unsprayed banana grown in large scale commercial farms, small farms, or backyard gardens on private property. The pathogen was isolated from individual leaves of banana and single ascospore cultures were obtained (Anonymous 1980; Fullerton and Olsen 1995). Dry leaf samples were examined to identify black streaks with fruiting bodies in the leaves. Pieces of leaf tissue $\left(20 \mathrm{~mm}^{2}\right)$ containing pseudothecia were cut from infected leaves using a sterile razor. Cut leaf tissue was surface sterilized, stapled to filter paper, and immersed in sterile distilled water for $30 \mathrm{~min}$. The filter papers were transferred to the lids of Petri dishes and left for 10 to $15 \mathrm{~min}$ to discharge ascospores over $3 \%$ water agar. Individual ascospores were removed from agar plates with a fine needle and transferred to potato dextrose agar. Mycelium of the pathogen was grown in potato dextrose broth. Total genomic DNA was extracted from mycelium using a standard cetyltrimethylammonium bromide protocol (Ristaino et al. 2001). DNA was diluted $1: 10$ or $1: 100$ ( 3 to $10 \mathrm{ng} / \mu \mathrm{l}$ ) for further use. Isolates were identified as $P$. fijiensis by polymerase chain reaction (PCR) using primers specific to the pathogen (Johanson 1995) and DNA sequencing.

DNA amplification and sequencing. Multilocus genotyping was done using six sequence-based markers from variable nuclear introns. Primers were developed by Robert et al. (2012a) utilizing the genome sequence of $P$. fijiensis (available at the JGI website: http://genome.jgi.doe.gov/Mycfi2/Mycfi2.home.html) and the fungal protein family database FunyBase (http://genome.jouy.inra.fr/ funybase/). Introns around genes for calmodulin (CaM), glyceraldehyde 3-phosphate dehydrogenase (G3PD), and translation elongation factor $1 \alpha$ (Tef1A), as well as three additional phylogenetically informative regions (Seq4, Seq8, and Seq12; FunyBase orthologous gene families FG855, FG552, and FG1000, respectively), were used. Primer sequences have been published previously (Robert et al. 2012a). PCR were performed in 50- $\mu$ l volumes for each sample. Each reaction contained $5 \mu \mathrm{l}$ of $10 \times$ PCR buffer (Genesee, San Diego, CA), $2.5 \mu$ of dNTP ( $2 \mathrm{mM}$ per nucleotide), $2 \mu \mathrm{l}$ of each $10 \mu \mathrm{M}$ forward and reverse primer, $1.8 \mu \mathrm{l}$ of $\mathrm{MgCl}_{2}(50 \mathrm{mg} / \mathrm{ml})$, $0.25 \mu \mathrm{l}$ of bovine serum albumen $(20 \mathrm{mg} / \mathrm{ml}), 0.2 \mu \mathrm{l}$ of Taq $(5 \mathrm{U} / \mu \mathrm{l})$ (Genesee), and 3 to $10 \mathrm{ng}$ of genomic DNA. The PCR program was modified from Ristaino et al. (2001) and Robert et al. (2012a), with an initial denaturation at $96^{\circ} \mathrm{C}$ for $2 \mathrm{~min} ; 30$ cycles of $94^{\circ} \mathrm{C}$ for $90 \mathrm{~s}$, $60^{\circ} \mathrm{C}$ for $2 \mathrm{~min}$, and $72^{\circ} \mathrm{C}$ for $3 \mathrm{~min}$; and a final extension of $72^{\circ} \mathrm{C}$ for 10 min. The PCR products were visualized on 1 to $1.6 \%$ agarose gels to verify amplification and PCR products were purified with Exosap-IT for PCR product cleanup (Affymetrix, Santa Clara, CA). Purified PCR products were sequenced directly in the forward and reverse directions either at the Genomic Sciences Laboratory at North Carolina State University (Raleigh) or GENEWIZ (Morrisville, NC).

Gene sequence analysis. A global populations sequence dataset for $P$. fijiensis was acquired from the Dryad Digital repository (Robert et al. 2012b) and analyzed in conjunction with the multilocus data generated from our research from collections from Costa Rica and Honduras. Sequences from each haplotype and gene region were submitted to GenBank (KY587714 to KY587736). Sequences were identified by country and by region (Southeast Asia, Oceania, Africa, and Americas), based on the grouping scheme of Robert et al. (2012a). Costa Rican and Honduran samples were designated as separate groups.

All statistical analyses of the nucleotide sequences were performed in SNAP Workbench, version 2.0 (Monacell and Carbone 2014). All sequences were aligned manually and edited using BioEdit (Hall 1999). Multiple sequence alignment was also performed in Clustal W (Thompson et al. 1994). Sequences were collapsed into unique haplotypes using SNAP Map (Aylor et al. 2006) after removing insertions and deletions from each of the aligned multilocus data sets and excluding infinite-sites violations. Resultant haplotype data sets were used to examine the overall support or conflict among the variable sites in the DNA sequence alignment. A site compatibility matrix was generated from each haplotype data set using SNAP Clade (Bowden et al. 2008). Compatibility matrices were used to examine compatibility and incompatibility among all variable sites, with any resultant incompatible sites removed from the data set. Data sets were also evaluated using Kwarg (Lyngsø et al. 2005) for estimating the minimum number of recombination events and constructing ancestral recombination graphs. Conflicting data partitions or putative recombinant haplotypes were excluded from further analyses, except when testing for population subdivision using Hudson's test statistics, because recombination increases the power of these tests (Hudson 2000; Hudson et al. 1992a,b). Nonrecombining data sets were collapsed into unique haplotypes excluding infinite-site violations using SNAP Map for all loci except G3PD, in which no recombination was detected. To investigate potential geographic sources of inoculum, the data were combined with the global data set of Robert et al. (2012b) for further analysis, unless otherwise noted.

Neutrality tests and population subdivision. The DNA sequences were analyzed using Arlequin (Excoffier and Lischer 2010). For each locus in each population, the population mean mutation rate per nucleotide site $\left(\theta_{\mathrm{w}}\right)$ was calculated using Watterson's $\theta$ (Watterson $1975)$, based on the number of segregating sites $(s)$ and the average pairwise nucleotide diversity $(\pi)$ (Tajima 1983), was estimated. Different tests of neutrality, including Tajima's D and Fu's Fs statistic (Fu 1997, Tajima 1989), were performed in order to determine whether the data were consistent with the expectations of the neutral model of molecular evolution.

Genealogical analysis. Maximum-likelihood trees were constructed utilizing the largest nonrecombining block for each of the six loci over the entire combined dataset in MEGA5, with 1,000 bootstrap replicates (Nei 1987; Saitou and Nei 1987; Tamura et al. 
2011). A maximum-likelihood tree was also generated using the largest nonrecombining block of all six loci concatenated. The Java program jModelTest 2.0 was used to determine the best model for each data set (Darriba et al. 2012; Guindon and Gascuel 2003).

Ancestral recombination graphs and coalescent analyses were generated using a concatenation of all six loci. This concatenated dataset was also used to generate coalescent trees using genetree (Griffiths and Tavaré 1994) after identifying the largest nonrecombining block as implemented in SNAP Workbench. Watterson's $\theta$ was used for estimations of the total population neutral mutation rate, and was estimated for each dataset using the program simple $\theta$ (Guindon and Gascuel 2003). Analyses were performed as panmictic populations with 10 million runs. The trees were generated 10 times, each using different random seed to evaluate convergence. The representative tree was chosen by examining the consensus of topology and mutation structure between all trees.

Haplotype analysis. The largest nonrecombining block for each locus was used to construct haplotype networks using the medianjoining method, as implemented in Network (v. 5.0.0.0; Fluxus Technology, Sudbury, UK), in order to examine relationships between haplotype diversity, haplotype distance, and geographic location. In addition, a haplotype network was constructed utilizing a concatenated dataset of all loci across all geographic regions. Populations were grouped by global region, as described by Robert et al. (2012a), with the exception of populations from Costa Rica and Honduras, which were designated as separate groups.

\section{RESULTS}

Nuclear sequence variability. In total, 3,182 nucleotides were sequenced from the Costa Rica and Honduran isolates, consisting of 523 nucleotides of the CaM locus, 469 nucleotides of the G3PD locus, 386 nucleotides of the Seq4 locus, 644 nucleotides of the Seq8 locus, 533 nucleotides of the Seq12 locus, and 627 nucleotides of the Tef1 A locus (Supplementary Table S2). When the largest nonrecombining sequence data partition was utilized, Seq12 (haplotypes $[\mathrm{h}]=14)$ and Tef1 A $(\mathrm{h}=12)$ loci had the most number of haplotypes across all populations. The lowest number of haplotypes across all populations were found in the G3PD $(h=6)$ and Seq4 $(h=7)$ loci. In addition, the G3PD locus had the lowest mean mutation rate $\left(\theta_{\mathrm{W}}\right)$. All loci were neutral based on Tajima's D and Fu's Fs. When the entire sequence was considered, the Seq12 locus had the greatest number of haplotypes $(\mathrm{h}=18)$ (Supplementary Table S3). When all loci were concatenated, 59 haplotypes were identified (Supplementary Table S4).

Populations from Southeast Asia had higher nucleotide diversity $(\pi)$ and mean mutation rates $\left(\theta_{\mathrm{W}}\right)$ across all loci than other populations. Conversely, populations from Africa were homogenous; only one haplotype was observed within each locus, except for $\mathrm{CaM}$ and Tef1A.

Two haplotypes were found within each locus for both Costa Rican and Honduran populations, with the exception of $\mathrm{CaM}$ and Seq8, in which only one haplotype was identified in each. All populations with more than one haplotype were determined to be neutral based on Tajima's D and Fu's Fs statistics across all loci, with the exception of Tajima's D for Seq8 in Indonesia.

Phylogeographic source of inoculum. Maximum-likelihood trees from concatenated sequence data from all loci revealed the presence of two clades, one containing isolates from Southeast Asia populations and one containing isolates from Southeast Asia, Oceania, Africa, and the Americas (Fig. 1). Only African populations formed an exclusive, monophyletic clade within the tree, which was a sister to a clade consisting of haplotypes from Southeast Asia. All haplotypes containing Costa Rican or Honduran isolates were found within a single clade that also included samples from Southeast Asia, Oceania, and the rest of the Americas.

Coalescent analyses from concatenated sequence data from all loci also showed the presence of two clades, one containing isolates from Southeast Asia and the other containing isolates from all populations (Fig. 2). Only African populations formed an exclusive, monophyletic clade, nested within a clade consisting solely of Southeast Asian haplotypes. Haplotypes containing Costa Rican and Honduran haplotypes were located in a clade that included representatives from Southeast Asia, Oceania, and other parts of the Americas, and was within a larger clade with a Southeast Asian origin.

Maximum-likelihood trees of individual loci were constructed and trends similar to those in the concatenated dataset were observed. Costa Rican and Honduran isolates were found in clades with Southeast Asian, Oceanian, and other American isolates for all loci. Honduran or Costa Rican isolates were also found in a clade that included African isolates for G3PD, Seq4, and Tef1A loci. Of these loci, shared haplotypes between Honduran, Costa Rican, and African isolates were found only for the G3PD locus. Within all other loci, isolates from Costa Rica and Honduras shared haplotypes with isolates of P. fijiensis from Southeast Asia, Oceania, and the Americas. Outside of the Americas, isolates from Papua New Guinea, Indonesia, Australia, Futuna, and Tonga shared haplotypes with Costa Rican and Honduran isolates across all six loci.

The ancestral recombination analysis indicated that all haplotypes from Costa Rican populations were generated through recombination. Four haplotypes were identified as exclusive to Costa Rican populations ( $\mathrm{H} 20, \mathrm{H} 23, \mathrm{H} 24$, and $\mathrm{H} 25)$. Two of the four haplotypes (H23 and H24) were generated through more recent recombination events. Two haplotypes were identified as exclusive to Honduran populations (H13 and H21), one of which (H21) was generated through a more recent recombination event.

Migration pathways into Costa Rica. Genetic substructuring and gene flow among populations of $P$. fijiensis for each locus was evaluated using the nearest-neighbor statistic. Costa Rican populations were subdivided from Southeast Asian, Oceanian, and African populations across all loci, with the exception of Oceania at G3PD. Costa Rican populations were also subdivided from other populations in the Americas based on Seq4 but gene flow between Costa Rican and other populations in the Americas was detected for all other loci. Gene flow was also detected between Costa Rica and Honduras for all loci examined. Gene flow was detected between Honduran and Oceanian populations from the CaM and G3PD loci, and between populations from Honduras and the Americas for all loci except Seq4.

A median-joining method was used to examine the largest nonrecombining block of sequence data for each locus as well as a concatenation of all loci. Costa Rican haplotypes shared close proximity with haplotypes from Southeast Asia when concatenated loci were examined (Fig. 3). A limited number of haplotypes were found in Costa Rican populations for each individual locus, and shared haplotypes with Oceania, Southeast Asia, the Americas, and Honduras were observed across all loci (Supplementary Fig. S3). In addition, Costa Rican populations shared a haplotype with populations from Africa for the G3PD locus. Haplotypes in Costa Rican populations were closest in similarity with haplotypes found in populations from Southeast Asia for the G3PD, Seq8, and Seq12 loci. Populations of $P$. fijiensis from Costa Rica clustered with populations from Southeast Asia and Oceania at the $\mathrm{CaM}$ and Tef1A loci. Costa Rican haplotypes of $P$. fijiensis were clustered with Southeast Asian and African haplotypes at the Seq4 locus.

\section{DISCUSSION}

Genetic diversity of Costa Rican populations. We collected an extensive number of isolates of $P$. fijiensis from Costa Rica over a 5-year period, primarily from commercial banana plantations and backyard gardens, and characterized the genetic structure of the pathogen population using the largest Costa Rican sample set to date. Our data revealed limited diversity within Costa Rican populations of P. fijiensis, with only a few haplotypes identified per locus. 
Low levels of genetic variability have also been observed in two previous studies that examined global populations and included a smaller number of isolates of the pathogen from Costa Rica (Carlier et al. 1996; Robert et al. 2012a). We know little about the genetic structure of the first introduction of the pathogen into Honduras, because original samples from the 1970s outbreaks are not available to test, but our data suggest that the pathogen diversity has changed little in the past 40 years since its first introduction.

Previous studies have suggested that the low level of diversity despite the presence of sexual reproduction may be the result of the founder effect (Carlier et al. 1996; Rivas et al. 2004; Robert et al. 2012a). P. fijiensis was first reported relatively recently in Central America in the 1970s, having arrived possibly through conidia on infected leaves used as packing material or through the importation of germplasm that moved into Costa Rica a few years later (Ploetz 2001; Stover 1978). The lack of rare alleles would keep the population diversity low, preventing extensive changes to current populations. In addition, regular treatment of commercial farms with fungicides and removal of necrotic tissue could reduce population diversity of $P$. fijiensis in these fields or shift populations toward asexual reproduction, which could limit the haplotype diversity (Rieux et al. 2013). However, ascospores from nearby private food crop gardens, which are not as rigorously sprayed or managed for black Sigatoka, may provide a source of new haplotypes (Rieux et al. 2013). Further sampling of nonsprayed private banana crops would need to be done to confirm or refute this hypothesis. Our data indicated that there were few haplotypes among fields in Costa Rica and they were shared regardless of production type.

Many of the isolates in our study came from commercial banana plantations that were sprayed heavily with fungicides and planted in monoculture. Other factors could affect pathogen population structure, including fungicide application, shade or sunlight, and host biodiversity. These factors were not examined in our work but could explain the low levels of diversity observed. Although the majority of outbreaks of $P$. fijiensis occur in commercial large-scale plantations, additional sampling of large plantations on smallholder farms or at higher altitudes may reveal additional haplotypes not previously observed here. The closely related sister species M. musicola, the causal agent of yellow Sigatoka, is typically found at higher altitudes (Churchill 2011; Gomes et al. 2013). P. fijiensis has also been found at increasingly higher elevations (Arzanlou et al. 2007). This shift may be due to changes in global temperature but may also reflect an adaptation of the pathogen to cooler climates, a question that needs further examination.

Examination of $P$. fijiensis on other hosts, such as plantain, may also reveal additional haplotypes. In Costa Rica, plantain are frequent in backyard gardens and are planted as a shade crop with coffee, and are not typically subject to the same levels of disease management as commercial banana plantations. These populations may also provide a refuge for rarer haplotypes of $P$. fijiensis. An

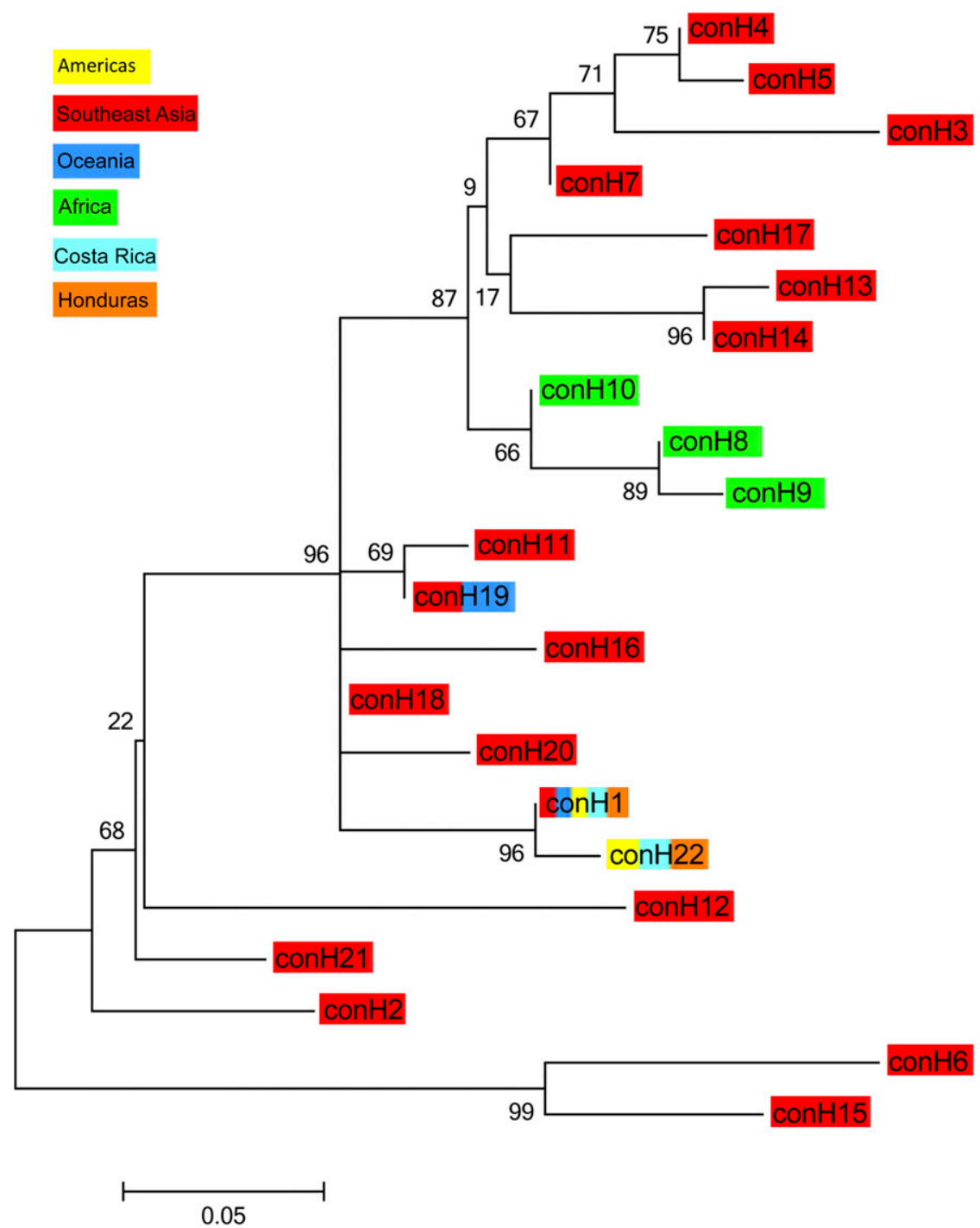

Fig. 1. Maximum-likelihood tree of global populations of Pseudocercospora fijiensis using six concatenated nuclear loci. Bootstrap values are based off of 1,000 repetitions. Haplotypes are coded by region and are based on the largest nonrecombining block. 
increase in diversity over a longer time period would be expected to occur through continued recombination and rare migration events (Churchill 2011). Our ancestral recombination graph data suggest that recombination is occurring in Central American populations of the pathogen; therefore, continued monitoring of population diversity over time and in different habitats within Costa Rica is warranted.
Sources of inoculum. Multilocus sequence data from populations of $P$. fijiensis from our study were compared with a previously published dataset of global populations to infer potential sources of inoculum within Costa Rican populations (Robert et al. 2012b). Although the dataset from Robert et al. (2012b) included some Costa Rican isolates, our study expands the Costa Rican dataset significantly to allow a more rigorous comparison of Costa Rican

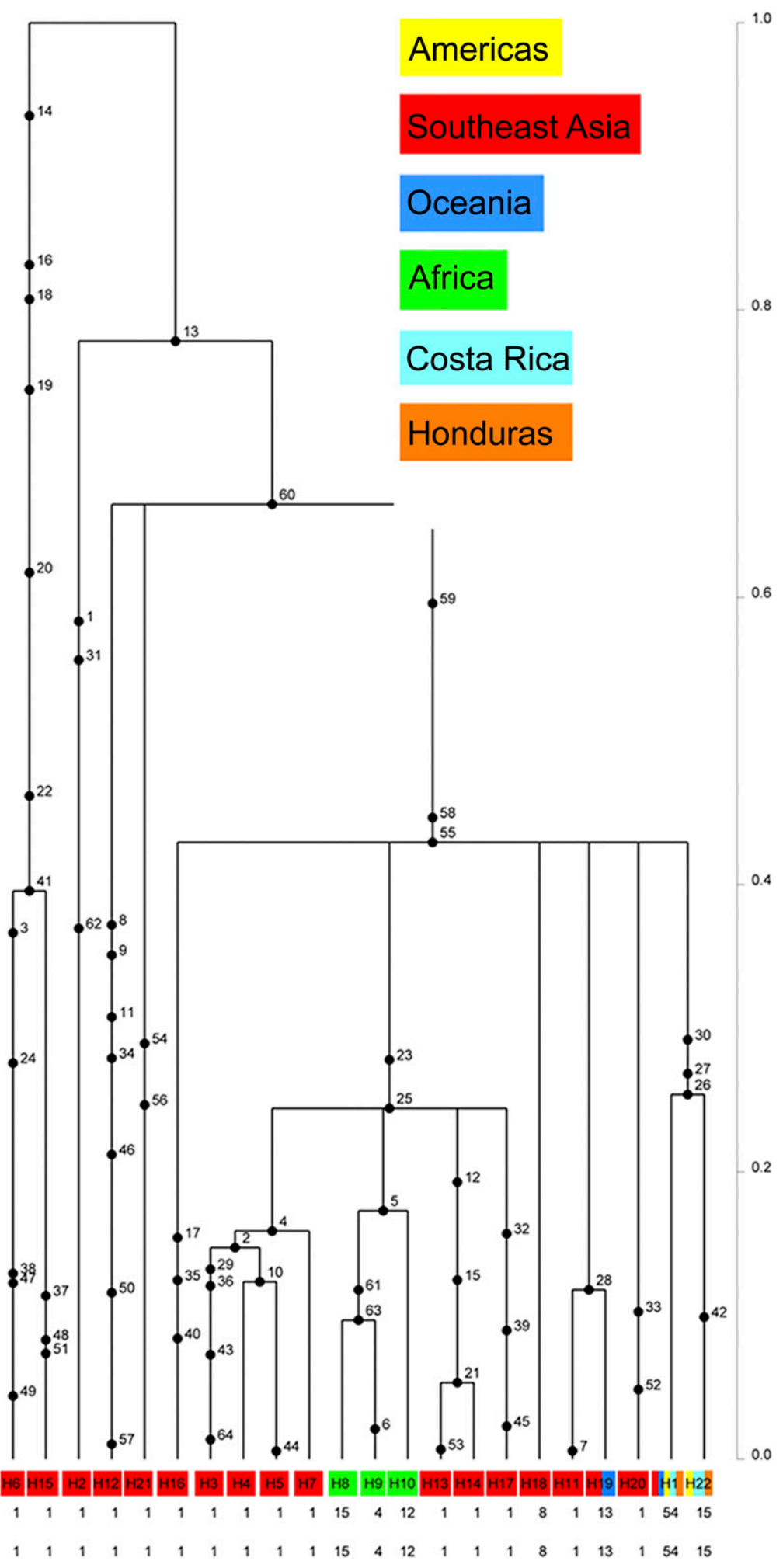

Fig. 2. Coalescent analysis of global populations of Pseudocercospora fijiensis in relation to Costa Rican and Honduran populations based on six concatenated loci. Tree was constructed using 50 million repeats and was repeated five times. Haplotypes are coded based on region and are based on the largest nonrecombining block. 
populations of the pathogen to global populations of the pathogen. Populations of $P$. fijiensis from Costa Rica shared genetic diversity with populations from other parts of the Americas, as well as Southeast Asia and Oceania. Costa Rican populations of $P$. fijiensis shared few haplotypes with populations of $P$. fijiensis from Africa, suggesting that an African introduction of the pathogen into Costa Rica is unlikely.

Examination of relationships between African haplotypes and haplotypes of $P$. fijiensis from other regions indicated that African haplotypes of the pathogen typically formed exclusive clades within larger clades of Southeastern Asian haplotypes. Thus, our data confirm the findings of others that African populations of $P$. fijiensis are likely the result of a single introduction out of Southeast Asia and have very little shared genetic diversity with Central American populations of $P$. fijiensis (Robert et al. 2012a).

Unlike African populations, Costa Rican populations of $P$. fijiensis do not form a single exclusive clade when compared with haplotypes of the pathogen from other regions. P. fijiensis haplotypes in Costa Rica were present in multiple clades that shared haplotypes with P. fijiensis from Southeast Asian and Oceania populations, suggesting a scenario involving more than one introduction of the pathogen. This may have occurred from either multiple introductions of the pathogen at a single location or introductions in multiple locations over time (Robert et al. 2012a). Further examination of common haplotypes of P. fijiensis between Costa Rica and Southeast Asia indicated that populations of $P$. fijiensis from Papua New Guinea and Indonesia shared haplotypes with Costa Rican populations of $P$. fijiensis across all loci. Haplotypes of $P$. fijiensis were also shared across all loci between Costa Rica and Oceania countries, including Australia, Futuna, and Tonga, suggesting that this region could also be a potential source of inoculum. However, historical evidence suggests that direct importation from an Oceanian region is unlikely.
With the exception of the importation of germplasm by the UFC, direct trade with countries in Oceania was minimal. However, UFC banana populations could have been moved outside of Honduras to other locations within Central America. An additional scenario is that the source of Costa Rican inoculum may be the result of admixture between populations from Southeast Asia, Oceania, and other parts of the Americas. This could possibly be due to admixture between Southeast Asia and Oceania lineages first, followed by admixture with or introduction into populations from the Americas, or due to importation at a common place.

We compared populations of $P$. fijiensis from Costa Rica with isolates collected from the site of first report of $P$. fijiensis in Honduras. Genetic diversity was shared between Costa Rican and Honduran populations of the pathogen at all loci, suggesting a strong association. In addition, significant levels of gene flow were detected between Costa Rican and Honduran populations of the pathogen in comparison with populations from other global regions. The proximity of the two countries suggests an alternate scenario, in which P. fijiensis spread from Honduras south to Costa Rica rather than via direct importation of banana from elsewhere in the world. The first report of P. fijiensis in Costa Rica was in 1979, approximately 7 years after the first report in Honduras (Halkett et al. 2010; Woods 1980), although it has been suggested that the pathogen was already present in the country the year before (Stover 1980). A previous published study using microsatellites examined Costa Rican populations of $P$. fijiensis, revealed low levels of genetic differentiation, and suggested continuous range expansion of the pathogen (Halkett et al. 2010). This was also supported by others who detected a decrease in genetic variability across Central America from Honduras into Venezuela (Robert et al. 2012a). Although another study using restricted fragment length polymorphism also saw higher levels of genetic diversity within Costa Rican and Honduran
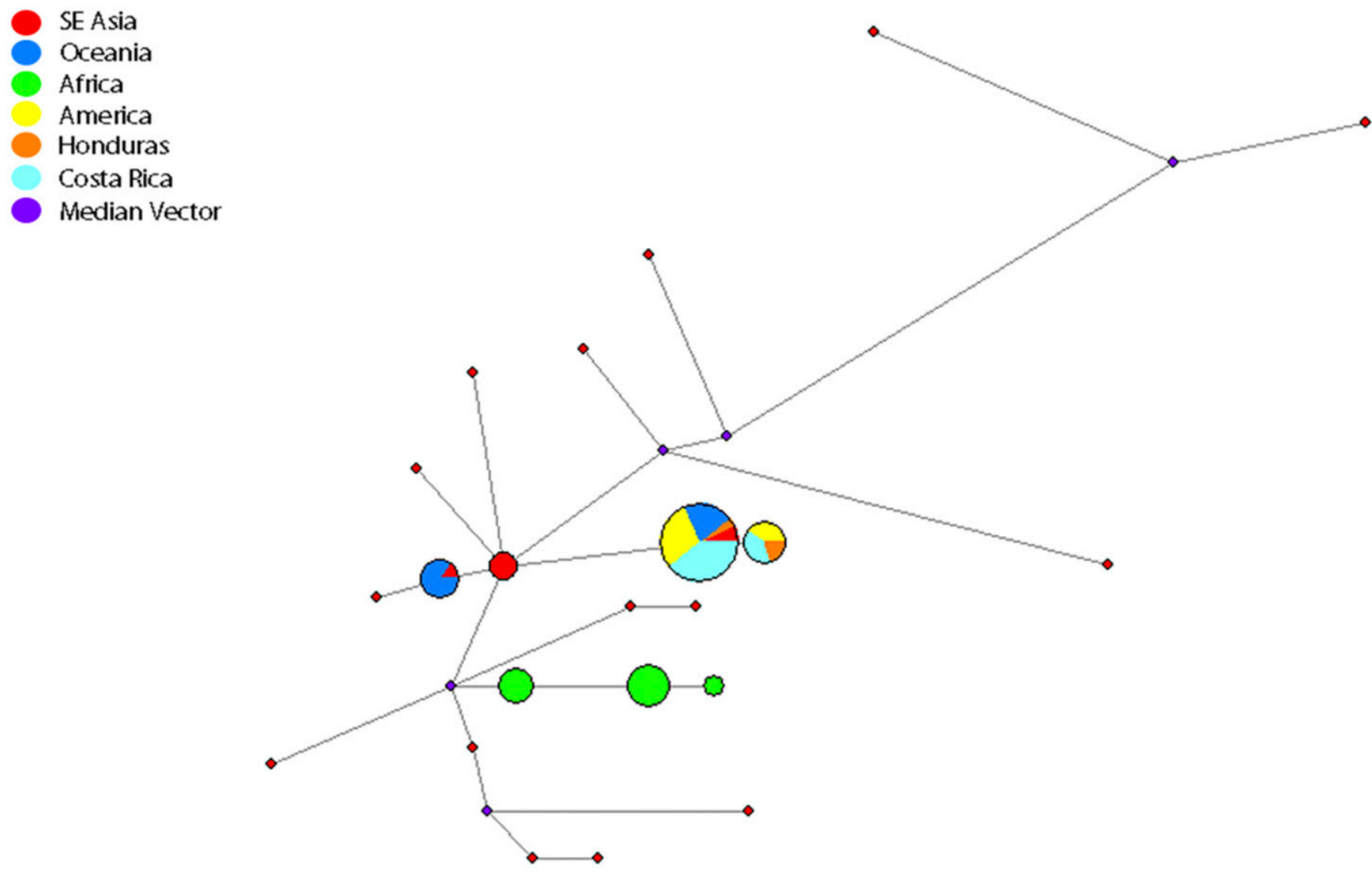

Fig. 3. Median joining networks for global populations of Pseudocercospora fijiensis based on the concatenation of six loci. Circles correspond to haplotypes and circle size is proportional to number of sequences. Branch length is proportional to number of mutations between haplotypes. Haplotypes are based on the largest nonrecombining block. 
populations in comparison with other American or Caribbean countries, it was suggested that the spread was more stochastic, consistent with the movement of infected host material (Rivas et al. 2004). It has been suggested that a likely trade route of $P$. fijiensis from Honduras to Costa Rica may have been through the transport of refuse banana and leaves from Honduras via truck (Stover 1980; Woods 1980). This mechanism was observed in the movement of disease from Guatemala to Mexico (Stover 1980). The use of banana leaves as packing material for produce may have also contributed to the spread of the pathogen, possibly acting as a source for multiple introductions, as well as the dispersal of ascospores over long distances by wind (Jones 2000).

The amount of shared genetic diversity between populations of $P$. fijiensis from Costa Rica, Honduras, and regions of Southeast Asia and Oceania may be due, in part, to the importation of banana germplasm from several hundred wild and cultivated banana lineages during the 1960s for use in a breeding program established in Honduras in 1959 (Robert et al. 2012; Rosales et al. 1999). The location of the breeding program was also the location of the first official report of P. fijiensis in Honduras (Robert et al. 2012a; Stover and Dickson 1976). Banana populations in the collection were imported primarily from the Western Pacific and Southeast Asia, including a large number of collections from Papua New Guinea, Malaysia, Indonesia, and the Philippines (Rosales et al. 1999). The banana breeding program may have been the source of multiple longdistance migration events of $P$. fijiensis into the Western Hemisphere. Although accession imports into Honduras were screened for disease off the coast of the country on the island of Utila, the farm where the collection was ultimately kept was also used for research on yellow Sigatoka, and a couple of acres were left unsprayed as a source of ascospores. In addition, intense trade was occurring between the Honduran islands and the coastal towns, providing a potential route for introduction to the mainland. Furthermore, as the banana industry in Central America expanded in the 1960s, technical personnel of the banana companies frequently traveled back and forth between regions in Southeast Asia and Central America, either on business or for sabbatical. Through one of these routes, black Sigatoka may have arrived and survived undetected among yellow Sigatoka in this region well before the time of first report (Jones 2000; Stover 1972, 1974; Stover and Dickson 1976,).

Additional sampling of the pathogen from Honduras, both at the site of first report and in surrounding areas, as well as from noncommercial smallholder farms at different elevations and environments (shade and full sun) on alternative hosts, may provide a clearer understanding of the impact of environment, host, and management practices on the genetic structure of the pathogen populations in the Central American region. Migration of $P$. fijiensis into Costa Rica via Honduras does not preclude the possibility of direct importation of the pathogen from outside Central America, and examination of historic trade routes or older samples may provide additional insight into the source of first introductions. Data from this study and historic written evidence suggest multiple introductions from a single site but broader sampling is needed before the "multiple introductions, multiple locations" scenario can be rejected.

\section{ACKNOWLEDGMENTS}

Funding for the Global Plant Health Scholars program was provided through the National Science Foundation International Research Experience for Students Grant Number 0966530. We thank Global Plant Health Scholars M. Lewis, J. Pope, M. Wyatt, and L. Bostic for assistance with collections; J. McGhee for technical assistance; M. Wyatt for assistance with data analysis; Z. Reyes, J. Melgar, and M. Rivera (FHIA Honduras) for providing samples from the original field of introduction in Honduras; G. Leandro and J. Gonzales (Dole Food Company) for providing access to the commercial plantations and funding for the research; and M. Blanco and M. Daub for providing summer internship coordination and lab opportunities for the Global Plant Health Scholars.

\section{LITERATURE CITED}

Abadie, C., Chilin-Charles, Y., Huat, J., Salmon, F., Pignolet, L., Carlier, J., Lescot, T., Cote, F., and Jenny, C. 2009. New approaches to select cultivars of banana with durable resistance to Mycosphaerella leaf spot diseases. Acta Hortic. 828:171-178.

Anderson, P. K., Cunningham, A. A., Patel, N. G., Morales, F. J., Epstein, P. R., and Daszak, P. 2004. Emerging infectious diseases of plants: Pathogen pollution, climate change and agrotechnology drivers. Trends Ecol. Evol. 19:535-544.

Anonymous. 1980. Black and yellow Sigatoka: Improved identification and management techniques. Dupont Latin America, Du Pont Agrichemicals, Coral Gables, FL.

Arzanlou, M., Abeln, E. C. A., Kema, G. H. J., Waalwijk, C., Carlier, J., de Vries, I., Guzmán, M., and Crous, P. W. 2007. Molecular diagnostics for the Sigatoka disease complex of banana. Phytopathology 97:1112-1118.

Aylor, D. L., Price, E. W., and Carbone, I. 2006. SNAP: Combine and Map modules for multilocus population genetic analysis. Bioinformatics 22: 1399-1401.

Bowden, L. C., Price, E. W., and Carbone, I. 2008. SNAP Clade and Matrix, version 2. Online publication. http://carbonelab.org/workbench

Carlier, J., Lebrun, M. H., Zapater, M. F., Dubois, C., and Mourichon, X. 1996. Genetic structure of the global population of banana black leaf streak fungus, Mycosphaerella fijiensis. Mol. Ecol. 5:499-510.

Churchill, A. 2011. Mycosphaerella fijiensis, the black leaf streak pathogen of banana: Progress towards understanding pathogen biology and detection, disease development, and the challenges of control. Mol. Plant Pathol. 12: $307-328$

Darriba, D., Taboada, G. L., Doallo, R., and Posada, D. 2012. jModelTest 2: More models, new heuristics and parallel computing. Nat. Methods 9:772.

Deighton, F. C. 1976. Studies on Cercospora and allied genera. VI. Pseudocercospora Speg., Pantospora Cif., and Cercoseptoria Petr. Mycol. Pap. 140:1-168

Desprez-Loustau, M. L., Robin, C., Buée, M., Régis, C., Garbaye, J., Suffert, F., Sache, I., and Rizzo, D. M. 2007. The fungal dimension of biological invasions. Trends Ecol. Evol. 22:472-480.

Etebu, E., and Young-Harry, W. 2011. Control of black Sigatoka disease: Challenges and prospects. Afr. J. Agric. Res. 6:508-514.

Excoffier, L., and Lischer, H. E. L. 2010. Arlequin suite ver. 3.5: A new series of programs to perform population genetic analyses under Linux and Windows. Mol. Ecol. Resour. 10:564-567.

FAO. 2014. Banana Market Review and Banana Statistics 2012-2013. Food and Agriculture Organization of the United Nations, Rome.

Fu, Y.-X. 1997. Statistical tests of neutrality of mutations against population growth, hitchhiking and background selection. Genetics 147:915-925.

Fullerton, R. A., and Olsen, T. L. 1995. Pathogenic variability in Mycosphaerella fijiensis Morelet, cause of black Sigatoka in banana and plantain. N. Z. J. Crop. Hortic. 23:39-48.

Gomes, L. I. S., Douhan, G. W., Biblano, L. B. J., Maffia, L. A., and Mizubuti, E. S. G. 2013. Mycosphaerella musicola identified as the only pathogen of the Sigatoka disease complex in Minas Gerais state, Brazil. Plant Dis. 97: 1537-1543.

Griffiths, R. C., and Tavaré, S. 1994. Ancestral inference in population genetics. Stat. Sci. 9:307-319.

Guindon, S., and Gascuel, O. 2003. A simple, fast and accurate method to estimate large phylogenies by maximum-likelihood. Syst. Biol. 52:696-704.

Halkett, F., Coste, D., Platero, G. G. R., Zapater, M. F., Abadie, C., and Carlier, J. 2010. Genetic discontinuities and disequilibria in recently established populations of the plant pathogenic fungus Mycosphaerella fijiensis. Mol. Ecol. 19:3909-3923.

Hall, T. A. 1999. A user-friendly biological sequence alignment editor and analysis program for Windows 95/98/NT. Nucleic Acids. Symp. Ser. 41:95-98.

Hudson, R. R. 2000. A new statistic for detecting genetic differentiation. Genetics 155:2011-2014.

Hudson, R. R., Boos, D. D., and Kaplan, N. L. 1992a. A statistical test for detecting geographic subdivision. Mol. Biol. Evol. 9:138-151.

Hudson, R. R., Slatkin, M., and Maddison, W. P. 1992b. Estimation of levels of gene flow from DNA sequence data. Genetics 132:583-589.

Johanson, A. 1995. Detection of banana leaf spot pathogens by PCR. EPPO Bull. 25:99-107.

Jones, D. R. 2000. Banana breeding for disease resistance. Pages 425-464 in: Diseases of Banana, Abacá and Enset. D. R. Jones, ed. CABI, Oxford.

Lyngsø, R. B., Song, Y. S., and Hein, J. 2005. Minimum recombination histories by branch and bound. Pages 239-250 in: Algorithms in Bioinformatics. R. Casadio and G. Myers, eds. Springer, Berlin. urnal")

Marciel Cordeiro, Z. J., and Pires de Matos, A. 2003. Impact of Mycosphaerella spp. in Brazil. Pages 91-97 in: Mycosphaerella leaf spot diseases of bananas: Present status and outlook. San José, Costa Rica. L. Jacome, 
P. Lepoivre, D. Marin, R. Ortiz, R. Romero, and J. V. Escalant, eds. 2nd Int. Workshop Mycosphaerella Leaf Spot Dis. Montpellier, France.

Monacell, T., and Carbone, I. 2014. Mobyle SNAP Workbench: A web-based analysis portal for population genetics and evolutionary genomics. Bioinformatics 30:1488-1490.

Nei, M. 1987. Molecular Evolutionary Genetics. Columbia University Press, New York.

Ortiz, R., and Vuylsteke, D. 1994. Inheritance of black Sigatoka disease resistance in plantain-banana (Musa spp.) hybrids. Theor. Appl. Genet. 89:146-152.

Ploetz, R. C. 2001. Black Sigatoka of banana: The most important disease of a most important fruit. Online publication. Plant Health Instruct. doi:10.1094/ PHI-I-2001-0126-02

Rieux, A., De Bellaire, D. L., Zapater, M. F., Ravigne, V., and Carlier, J. 2013. Recent range expansion and agricultural landscape heterogeneity have only minimal effect on the spatial genetic structure of the plant pathogenic fungus Mycosphaerella fijiensis. Heredity 110:29-38.

Ristaino, J. B., Groves, C. T., and Parra, G. R. 2001. PCR amplification of the Irish potato famine pathogen from historic specimens. Nature 411:695-697.

Rivas, G.-G., Zapater, M.-F., Abadie, C., and Carlier, J. 2004. Founder effects and stochastic dispersal at the continental scale of the fungal pathogen of bananas Mycosphaerella fijiensis. Mol. Ecol. 13:471-482.

Robert, S., Ravigné, V., Zapater, M.-F., Abadie, C., and Carlier, J. 2012a. Contrasting introduction scenarios among continents in the worldwide invasion of the banana fungal pathogen Mycosphaerella fijiensis. Mol. Ecol. 21:1098-1114.

Robert, S., Ravigné, V., Zapater, M.-F., Abadie, C., and Carlier, J. 2012b. Data from: Contrasting introduction scenarios among continents in the worldwide invasion of the banana fungal pathogen Mycosphaerella fijiensis. Online publication. Dryad Digital Repository.

Rosales, F. E., Tripon, S. C., and Cerna, J., eds. 1999. A Tribute to the Work of Paul H. Allen: A Catalogue of Wild and Cultivated Bananas. INIBAP/CIID/ EARTH, Montpellier, France.
Saitou, N., and Nei, M. 1987. The neighbor-joining method: A new method for reconstructing phylogenetic trees. Mol. Biol. Evol. 4:406-425.

Stover, R. H. 1972. Banana, Plantain and Abaca Diseases. Commonwealth Agricultural Bureaux, Kew, Surrey, UK.

Stover, R. H. 1974. Black Sigatoka. United Brands Company, Division of Tropical Research, Annual Report (1973) 3, San Pedro Sula, Honduras.

Stover, R. H. 1978. Distribution and probable origin of Mycosphaerella fijiensis in Southeast Asia. Trop. Agric. (Trinidad) 55:65-68.

Stover, R. H. 1980. Sigatoka leaf spots of bananas and plantains. Plant Dis. 64: $750-756$

Stover, R. H., and Dickson, J. D. 1976. Banana leaf spot caused by Mycosphaerella musicola and P. fijiensis var. difformis: A comparison of the first Central American epidemics. FAO Plant Prot. B. 24:36-42.

Tajima, F. 1983. Evolutionary relationship of DNA sequences in finite populations. Genetics 105:437-460.

Tajima, F. 1989. Statistical method for testing the neutral mutation hypothesis by DNA polymorphism. Genetics 123:585-595.

Tamura, K., Peterson, D., Peterson, N., Stecher, G., Nei, M., and Kumar, S. 2011. MEGA5: Molecular evolutionary genetics analysis using maximum likelihood, evolutionary distance, and maximum parsimony methods. Mol. Biol. Evol. 28:2731-2739.

Thompson, J. D., Higgins, D. G., and Gibson, T. J. 1994. CLUSTAL W: Improving the sensitivity of progressive multiple sequence alignment through sequence weighting, position specific gap penalties and weight matrix choice. Nucleic Acids Res. 22:4673-4680.

Watterson, G. A. 1975. On the number of segregating sites in genetic models without recombination. Theor. Popul. Biol. 7:256-276.

Woods, T. L. 1980. The black Sigatoka situation in Costa Rica. In: Proc. Sigatoka Workshop, La Lima, Honduras. D. T. Krigsvold and T. L. Woods, eds. Division of Tropical Research, United Fruit Co., La Lima, Honduras. 